\title{
Calidad de la dieta y cercanía a locales de comida rápida
}

\author{
Food quality and population fast-food restaurants accessibility
}

Boone-Heinonen J. y col. Arch Intern Med. 2011;171(13):1162-1170

\section{Objetivo}

Establecer la vinculación entre la proximidad a distintos tipos de fuentes de alimentos y la calidad de la dieta, según el nivel de ingresos.

\section{Diseño, lugar y pacientes}

Análisis de la cohorte CARDIA (Coronary Artery Risk Development in Young Adults) con población de base en Birmingham, Chicago, Minneapolis, y Oakland (EE.UU). Esta incluyó 5.115 personas entre 18 y 30 años de edad, analizadas a los dos, siete, diez y 15 años del inicio del programa, con una tasa de retención* del $90 \%, 86 \%, 81 \%$ y $79 \%$ en cada oportunidad de control.

\section{Evaluación de factores pronósticos}

Se identificaron los locales de comida rápida (CR), supermercados y pequeñas tiendas de alimentos existentes en radios concéntricos a la vivienda de cada participante de hasta $1 \mathrm{~km}$., 1 a 2,99 km., 3 a 4,99 km., y 5 a 8,05 km de distancia. Se encuesto y evaluó el consumo semanal de comida rápida, de frutas y hortalizas, mediante el Índice de Calidad de la Dieta (Diet Quality Index).

\section{Medición de Resultados Principales}

Los efectos asociados a la disponibilidad de distintas fuentes de alimentos fueron estimados por niveles de ingreso utilizando modelos de regresión linear de efectos fijos*, ajustados* por edad, variación con el tiempo, ingresos, estado civil, número de hijos, y el porcentaje de personas cuyos ingresos cayeron por debajo del $150 \%$ del nivel federal de pobreza.

\section{Resultados Principales}

La relación entre la disponibilidad de CR y el consumo individual de estos alimentos difirió significativamente según el nivel de ingresos $(P<0,05)$. Solo entre varones de bajos ingresos, un $1 \%$ de aumento en la disponibilidad de comida rápida a $1 \mathrm{~km}$ y de 1 a 2,99 km se relacionó con aumentos en el consumo de CR de un $0,13 \%$ y $0,34 \%$ respectivamente. A mayores distancias no hubo asociación significativa.

La disponibilidad de supermercados y tiendas de alimentos en el vecindario no se relacionó con la calidad de la dieta, para ningún nivel de ingresos.

\section{Conclusiones}

Los resultados obtenidos no respaldan algunas asunciones de política sanitaria (EUA) orientadas a mejorar la disponibilidad geográfica de fuentes de alimentos saludables. Este hallazgo alienta el mayor énfasis en estrategias alternativas, como el subsidio para alimentos específicos.

Por otra parte la reducción de locales de comida rápida en radios menores a $3 \mathrm{~km}$ de zonas de bajos ingresos podría producir reducciones en el consumo de ese tipo de dieta, en personas jóvenes y de mediana edad.

Palabras claves: Cohorte, dieta, bajos ingresos, CARDIA, comida rápida. Key words: Cohort, diet, low income, CARDIA, fast food Fuente de financiamiento: No referida.

\section{Comentario}

El proyecto CARDIA es un estudio iniciado en 1985, que en el presente trabajo es analizado desde la óptica de las políticas de salud propuestas por la actual administración gubernamental en EE.UU. Otros hallazgos del programa han vinculado componentes del estilo de vida, cambios en el peso, variaciones en la frecuencia de consumo de CR, e insulino resistencia'.

El problema del consumo de CR ya ha sido reportado con anterioridad: $37 \%$ de los adultos y $42 \%$ de los niños norteamericanos acceden habitualmente a CR, con su consecuente mayor ingesta energética, de grasas (especialmente saturadas), sodio, y un menor consumo de vitaminas ( $\mathrm{A}$ y $\mathrm{C}$ ), leche, frutas y vegetales ${ }^{2}$, situación que constituye un desafío para la salud pública contemporánea.

Existen resultados sugerentes de que el consumo está influido por grupos sociales de referencia, y la satisfacción de necesidades inmediatas (sabor, conveniencia), minimizando las preocupaciones de las personas sobre los riesgos para la salud a largo plazo $^{3}$. La vinculación entre cercanía física a la oferta (caminatas menores a 10 minutos) y consumo de CR ha sido reportada recientemente por otros investigadores, enfatizando la importancia de la reducción en el consumo de alimentos ricos en energía y bebidas azucaradas en la prevención de la obesidad, a través de ofrecer otras alternativas alimentarias, reducir la exposición (acceso), o realizar acciones educativas 4 .

Los autores del artículo que hoy comentamos, en vista de los resultados obtenidos, enfatizan sobre la conveniencia de promover la disponibilidad de alimentos saludables, más que insistir en políticas de restricción del acceso a CR.

Probablemente estrategias similares serían adecuadas en nuestro medio, donde resultan previsibles las dificultades para imponer y fiscalizar limitaciones a la actividad comercial, y en cambio políticas de promoción y expansión del mercado de alimentos saludables podrían resultar más efectivas, bajo modalidades y en plazos que podrían ser motivo de investigación.

Javier Oscar Vilosio [ Departamento de Salud Pública del Instituto Universitario del Hospital Italiano de Buenos Aires. javier.vilosio@ hospitalitaliano.org.ar ]

Vilosio JO. Calidad de la dieta y cercanía a locales de comida rápida. Evid Act Pract Ambul. Jul-Sep 2011;14(3):103. Comentado de: BooneHeinonen y col. (The CARDIA Study). Fast Food Restaurants and Food Stores. Longitudinal Associations With Diet in Young to Middle-aged Adults: The CARDIA Study. Arch Intern Med. 2011;171(13):1162-1170. PMID: 2174701

Referencias
1. Pereira MA, y col. Fast-food habits, weight gain, and insulin resistance (the CARDIA study): 15-year prospective analysis. The Lancet 2005;365(9453):36-42.

2 Paeratakul S, y col. Fast-food consumption among US adults and children: Dietary and nutrient intake profile, Joumal of the American Dietetic Association 2003;103(10):1332-1338.

3 Dunn Kl, y col. Determinants of fast-food consumption. An application of the Theory of Planned Behaviour. Appetite 2011;57(2):349-57.

4 Hearst MO, y col. Urban v. suburban perceptions of the neighbourhood food environment as correlates of adolescent food purchasing. Public Health Nutr. 2011;23:1-8. 